\title{
Phase-Sensitive Amplification and Regeneration of Dual-Polarization BPSK without Polarization Diversity
}

\author{
Abel Lorences-Riesgo ${ }^{(1)}$, Carl Lundström ${ }^{(1)}$, Fabrizio Chiarello $^{(2)}$, \\ Magnus Karlsson ${ }^{(1)}$, and Peter A. Andrekson ${ }^{(1)}$
}

(1) Photonics Laboratory, Dept. of Microtechnology and Nanoscience (MC2), Chalmers University of Technology, Gothenburg, SE412-96, Sweden, lorences@chalmers.se

${ }^{(2)}$ Dept. of Information Engineering, University of Padova, Via G. Gradenigo 6/B, Padova 35131, Italy

Abstract We analyze the performance of a degenerate vector PSA-based receiver and compare to an EDFA-based receiver, achieving an about $1 \mathrm{~dB}$ sensitivity improvement. We also assess the phase regeneration provided by the vector PSA when the signal phase is degraded.

\section{Introduction}

Phase-sensitive amplifiers (PSAs) have many potential applications such as noiseless amplification $^{1}$ and regeneration of phaseencoded signals ${ }^{2}$. These applications have been demonstrated with implementations based on either $\chi^{(2)}$ materials $^{3}$ or $\chi^{(3)}$ materials $^{1,2}$ and with different single-polarization (SP) modulations formats such as binary phase-shift keying $(B P S K)^{2}$, quadrature phase-shift keying $(Q P K)^{1}$ and 16-level quadrature amplitude modulation (QAM) ${ }^{3}$.

However, not much attention has been given for phase-sensitive (PS) amplification of dualpolarization (DP) signals. PS amplification of DP signals has been achieved by polarization diversity schemes ${ }^{3}$ with increased complexity of the system. As previously discussed ${ }^{4}$ and as we will show in this paper, a vector PSA can perform PS amplification of DP signals without polarization diversity. To the best of our knowledge, the performance of vector PSAs has up to date only been assessed with continuous wave $(\mathrm{CW})$ signals $^{5}$.

In this work, we demonstrate for the first time PS amplification of a DP-BPSK signal in a degenerate vector PSA. We assess the PSA performance working as a pre-amplifier as well as a phase regenerator.

Principle of operation: amplification of DPBPSK signals with a degenerate vector PSA

The polarization diagram for a degenerate vector PSA is shown in Fig. 1(a). The pumps, $P_{1}$ and $P_{2}$, are cross-polarized and the signal, $S_{x}$, and idler, $S_{y}$, are the components of the degenerate wave, $S$, in the pump polarization states described by the Jones vectors $\bar{x}$ and $\bar{y}$.

The relation between the input and output fields can be described by ${ }^{6}$ :

$$
\left[\begin{array}{l}
S_{\text {out }, x} \\
S_{\text {out }, y}^{*}
\end{array}\right]=\left[\begin{array}{cc}
\mu & v \\
v^{*} & \mu^{*}
\end{array}\right]\left[\begin{array}{l}
S_{i n, x} \\
S_{i n, y}^{*}
\end{array}\right]
$$

where $\mu$ and $v$ are the coefficients of the

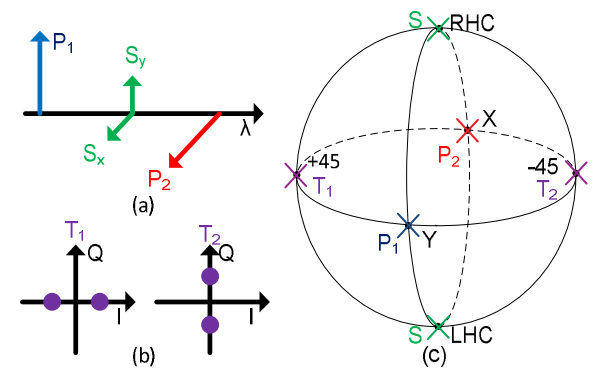

Fig. 1: (a) Polarization diagram of a degenerate vector PSA. (b) Constellation and (c) Poincaré sphere diagrams showing the relation between the pump and polarization tributaries for PS amplification of PM-BPSK with a degenerate vector PSA.

matrix transformation, with the phase-insensitive equals $|\mu|^{2}=1+|v|^{2}$. The PS gain can be determined as $^{5}$ :

$$
G(\alpha, \phi)=|\mu|^{2}+|v|^{2}+2|\mu \|| v \mid \sin (\alpha) \cos (\phi)
$$

where $\alpha$ is the polarization angle between the signal and the pumps (in Stokes space) and $\phi=\phi_{P 1}+\phi_{P 2}-\phi_{S, x}-\phi_{S, y}$ is the relative phase between the pumps, signal and idler.

The degenerate vector PSA can only PS amplify modulation formats for which the relative phase, $\phi$, is independent of the constellation points.

The vector PSA can PS amplify DP-BPSK signals under certain polarization conditions. The DP-BPSK signal can be expressed as:

$$
\bar{S}=a \bar{T}_{1}+b e^{j \theta} \bar{T}_{2}
$$

where $\bar{T}_{1}$ and $\bar{T}_{2}$ are two orthogonal Jones vectors defining the polarization of each individual polarization tributary and $\theta$ is the relative phase between them. The modulation symbol are defined by $a$ and $b$ which can take values of \pm 1 . This signal can take two antipodal instantenous states of polarization (SOPs) with two different global phases. In order to achieve PS amplification, the two possible signal instantanous SOPs have $\alpha=90^{\circ}$. Moreover, each one of the polarization tributaries should be amplified regardless of the data on the other 


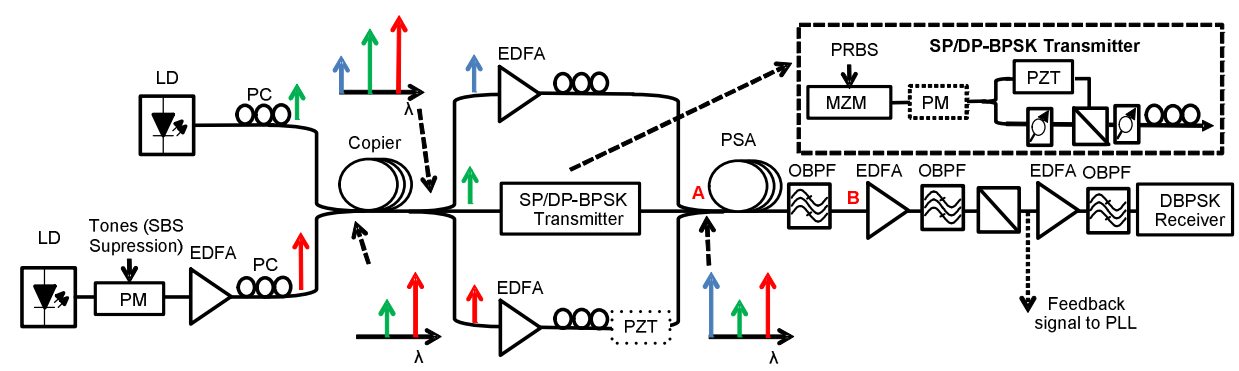

Fiq. 2: Experimental setup. The acronvms are defined in the text.

one. These two conditions are fullfilled when the tributary polarization $\left(\bar{T}_{1}\right.$ and $\left.\bar{T}_{2}\right)$ correspond, for example, to $\bar{T}_{1}=(\bar{x}+\bar{y}) / \sqrt{2}$, $\bar{T}_{2}=(\bar{x}-\bar{y}) / \sqrt{2}$ with $\theta=\pi / 2$. With this choice, the relative phase only takes a single value since $\phi_{S, x}+\phi_{S, y}$ is independent of the data.

The constellation diagram of the PM-BPSK signal which fulfills the previous condition is shown is Fig. 1(b). The polarization of the tributaries is linear at $+45^{\circ}$ and $-45^{\circ}$ with one channel modulated in the in-phase component and the other in quadrature. However, the net polarization of the signal $S$ will be left- or righthand circular, as plotted on the Poincaré sphere in Fig. 1(c). Note that this diagram can be rotated around the center without losing validity.

We should point out that the suggested DPBPSK format can be interpreted as a conjugated pair of QPSK signals, one in each polarization, similar to what was used by Liu et al. ${ }^{7}$ with a different purpose, to demonstrate nonlinear compensation.

\section{Pre-Amplifier}

We start by demonstrating the DP-PSA as a pre-amplifier in a back-to-back configuration. The experimental setup is shown in Fig. 2. We injected two CWs generated by two different laser diodes (LDs) with wavelengths of 1554 and $1558.6 \mathrm{~nm}$ into the copier ${ }^{1}$ where a third wave at wavelength of $1549.8 \mathrm{~nm}$ was created. The copier consisted of a $300 \mathrm{~m}$ long highly non-linear fiber (HNLF). After the copier, the signal and pumps were divided into three different paths by using wavelength-division multiplexing couplers which removed any other signals at different wavelengths than previously mentioned. The pumps, waves at 1549.8 and $1558.6 \mathrm{~nm}$, were amplified and filtered. The signal was modulated with a Mach-Zehnder modulator (MZM) at $10 \mathrm{Gbit} / \mathrm{s}$ with a pseudorandom binary sequence (PRBS) of length $2^{15}-1$ and divided into two different branches that had fiber length difference corresponding to $\sim 10$ ns for decorrelation. In one branch we included a piezoelectric transducer (PZT). Since stable instantaneous signal SOP was required, but we lack a DP-MZM, two PZTs would be required to compensate for the relative phase and track the signal polarization. The second PZT would be placed as indicated by the dotted line in the lower branch. With only one PZT, we could only make sure that one of the data channels was amplified while the other was randomly varying between PS amplification and attenuation due to phase drift. A variable optical attenuator enabled us to switch between a SP- or DP-BPSK signal. The DP-BPSK was created by combining both decorrelated tributaries with a polarization-beam splitter. The modulated signal was combined with the pumps before the PSA which consisted of a $150 \mathrm{~m}$ long HNLF. Each pump power was $\sim 30 \mathrm{dBm}$ which provided a PSA gain of $\sim 9 \mathrm{~dB}$. We controlled each wave SOP at the PSA input with polarization controllers (PCs) and tracked that the pumps were orthogonal by a polarimeter connected to a monitor port. After the PSA, an optical band-pass filter (OBPF) with $\sim 1.3 \mathrm{~nm}$ bandwidth filtered the signal. Then, the signal was amplified by an erbium-doped fiber amplifier (EDFA), filtered to remove out-of-band noise with a $\sim 2 \mathrm{~nm}$ OBPF and split into both polarization tributaries. We tapped the tributary corresponding to the branch of the PZT and detected to feedback the phase-locked loop (PLL) circuit. This tributary was also amplified by an EDFA and filtered by a $\sim 0.3 \mathrm{~nm}$ OBPF. The differential-BPSK (DBPSK) receiver consisted of a delay interferometer and balanced detector before the bit-error rate (BER) counter.

Fig. 3 shows the sensitivity for both SP- and DP-BPSK signals. The received signal power was measured at the input of the PSA, point $A$ in Fig. 2. For comparison, we include the sensitivity curve bypassing the PSA and measuring the received power at the input of the EDFA, point $B$. Using the PSA, the sensitivity at BER of $10^{-9}$ was $\sim-40 \mathrm{dBm}$ for the SP-BPSK signal and $\sim-37 \mathrm{dBm}$ for the DP-BPSK signal. The curves show a $\sim 1 \mathrm{~dB}$ sensitivity improvement using the PSA with regard to the EDFA case. The sensitivity improvement is about the same for both formats since there was no extra penalty due to the second polarization channel apart from the $3 \mathrm{~dB}$ power difference. This demonstrates that the scheme is not affected by the presence of the second channel 


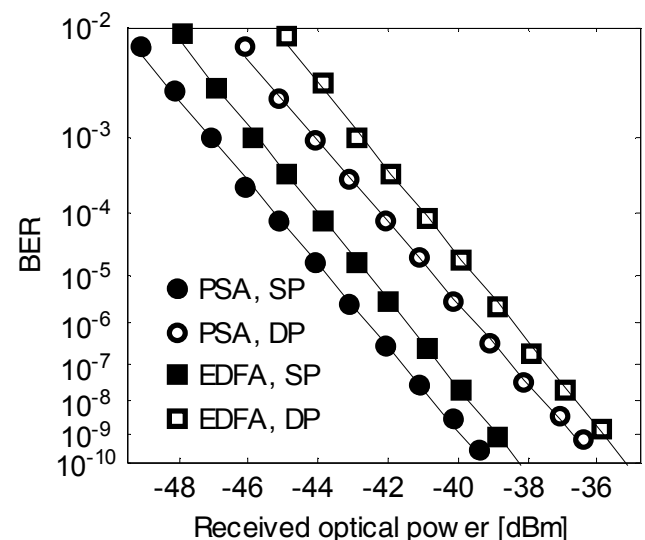

Fig. 3: Receiver sensitivity for SP-BPSK and DP-BPSK signals when using the PSA or EDFA as preamplifier.

which was not locked in phase and it was either amplified or attenuated according to the relative phase between it and the pumps. Simultaneous amplification of both tributaries can be achieved by either a second PLL or stable instantaneous SOP. The improvement is limited by the low net gain, $8.2 \mathrm{~dB}$, between the PSA and EDFA (including OBPF loss). The noise figure (NF) of the EDFA was $\sim 4.9 \mathrm{~dB}$. Taking into account the $\sim 1 \mathrm{~dB}$ improvement and gain, the expected PSA $\mathrm{NF}$ is $\sim 3 \mathrm{~dB}$. The presence of higher-order idlers deteriorated the PSA performance. It would be possible to remove the higher-order idler by appropriate dispersion mapping and pump wavelength selection. We placed the pumps with a separation of only $\sim 9 \mathrm{~nm}$ to minimize the polarization-mode dispersion effects. Moreover, the pump phase modulation in order to suppress stimulated Brillouin scattering can induce a certain penalty in the PSA performance.

\section{Phase Regeneration}

We also demonstrated the phase regeneration performance of the degenerate vector PSA. In this case, a phase modulator (PM) driven by thermal noise with bandwidth up to $4 \mathrm{GHz}$ and RMS swing of $\sim \pi / 2$ rad was included after the MZM. We analyzed the constellation before and after the PSA with a modulation analyzer (EXFO PSO-200). The obtained constellations are plotted in Fig. 4. As depicted, the phase was regenerated in both polarizations and as expected the phase fluctuations were converted into an amplitude noise. Due to the aforementioned lack of a second PLL, the samples shown were taken when the randomly drifting tributary was maximally amplified. The regeneration was not hampered by the presence of two tributaries. The results show that the proposed PSA is suitable as phase regenerator of DP-BPSK without the need of polarization diversity. Further studies should be performed to assess its performance as amplitude regenerators in gain saturation and the crosstalk

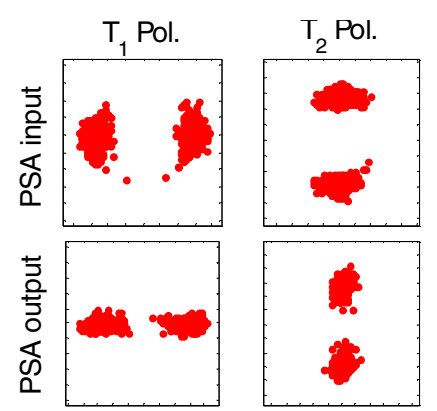

Fig. 4: Constellation diagrams for the phase degraded DP-BPSK signal before and after the PSA.

between tributaries since the presence of a strong tributary would deplete the pumps and lowered the gain over the other tributary ${ }^{4}$.

\section{Conclusions}

We have demonstrated the use of a degenerate vector PSA as amplifier and phase-regenerator of PM-BPSK signals. To the best of our knowledge, this is the first experimental demonstration of PS amplification of DP data without using polarization diversity. As a preamplifier, the scheme achieved $\sim 1-\mathrm{dB}$ improvement with regard to the EDFA case. There was no significant penalty due to the presence of both polarization components. The presence of higher-order idlers and the low gain limited the PSA performance. We expect that the performance could be enhanced by suitable design of the HNLF dispersion.

As a regenerator, we show that after degrading the signal with phase noise, both tributaries were phase-regenerated. This demonstrates the potential of the vector PSA as a phase regenerator of PM-BPSK signals without the additional complexity of polarizationdiversity schemes.

\section{Acknowledgements}

This work is supported by the European Research Council under grant agreement ERC-2011-AdG- 291618 PSOPA. We thank OFS Denmark and Sumitomo Electric Industries, Ltd. for providing HNLFs and EXFO for the loan of the modulation analyzer.

\section{References}

[1] Z. Tong et al., "Ultralow Noise, Broadband PhaseSensitive Optical Amplifiers, and Their Applications," JSTQE, 18, 1016 (2012).

[2] R. Slavík et al., "Coherent All-Optical Phase and Amplitude Regenerator of Binary Phase-Encoded Signals," JSTQE, 18, 859 (2012).

[3] T. Umeki, et al., "First Demonstration of High-Order QAM Signal Amplification in PPLN-Based Phase Sensitive Amplifier," Opt. Exp., 23, 2473 (2014).

[4] M. E. Marhic, "Polarization Independence and PhaseSensitive Parametric Amplification," J. Opt. Soc. Am. B., 28, 2685 (2011).

[5] A. Lorences-Riesgo et al., "Demonstration of Degenerate Vector Phase-Sensitive Amplification," ECOC 2013, We.3.A.3, (2013).

[6] C. J. McKinstrie and S. Radic, "Phase-Sensitive Amplification in a Fiber," Opt. Exp., 12, 4973 (2004).

[7] X. Liu et al., " Phase-Conjugated Twin Waves for Communication beyond the Kerr Nonlinearity Limit" Nat. Photonics, 7, 560 (2013). 